\title{
The use of capsaicin cream in a case of erythromelalgia
}

\author{
K.A. Muhiddin, I.W. Gallen, S. Harries and V.R. Pearce \\ Department of General Medicine, Royal Devon and Exeter Hospital, Exeter, UK
}

\begin{abstract}
Summary: We present a case of erythromelalgia in a 68 year old lady who responded, within 48 hours, to a twice daily topical application of capsaicin cream $0.025 \%$. Capsaicin cream was stopped after 2 months, and 6 months later the patient continued to have the symptomatic relief she experienced initially.
\end{abstract}

\section{Introduction}

Erythromelalgia is a rare syndrome characterized by pain, redness and increased skin temperature of one or more extremities. It was first described by Mitchell in $1878 .{ }^{1}$ Symptoms are characteristically exacerbated by dependency or heat exposure, and relieved by elevation or cooling of the affected limb. ${ }^{2,3}$ Capsaicin is a new topical medication for neuralgia. It is thought that capsaicin accentuates the release of substance $P$ from sensory nerve fibres and after repeated applications depletes the neurones of substance $P$, which is an important mediator of nociceptive impulses. ${ }^{13,14}$ It is not clear what the underlying mechanism of pain in erythromelalgia is, but it is possible that substance $P$ is involved. We report an intractable case of erythromelalgia and its successful response to capsaicin cream.

\section{Case report}

A 68 year old woman presented with a 10-month history of severe burning pain in the anterior surface of both lower legs and feet. Her symptoms started 3 months after she had had a lumbar spinal fusion operation. She developed tingling in the feet, which spread to involve the legs associated with burning pain, redness and foot swelling. She described hyperaesthesiae to light stimuli, although she was able to tolerate firmer handling. The pain was predominantly worse when walking leading to total immobility.

On examination there was intense erythema of both lower legs and feet, which did not blanch on pressure, and turned a purpliș hue when dependent. Her feet were warm and peripheral pulses

Correspondence: K.A. Muhiddin, Ph.D., M.R.C.P., Medicine for the Elderly, Derby City General Hospital, Uttoxeter Road, Derby DE22 3NE, UK.

Accepted: 3 November 1993 were full. Straight leg raising was limited to about $45^{\circ}$ by back discomfort, although movement of her lower limb joints was reasonable. Erythromelalgia was diagnosed and aspirin $75 \mathrm{mg}$ daily and propranolol $80 \mathrm{mg}$ twice daily were commenced. Two months later, no appreciable symptomatic relief was observed. In addition, mild cardiac failure was precipitated by the beta blocker.

Extensive laboratory investigations were performed at presentation. Full blood count, erythrocyte sedimentation rate, urea, creatinine and electrolytes, liver function tests and thyroid function tests were all normal. Autoimmune profile and rheumatoid factor were negative. Protein electrophoresis and immunoglobulin levels were normal.

Capsaicin cream $0.025 \%$ was applied topically to a small area in the right lower leg, and an inert cream (E-45) was applied to the left lower leg as a control. This was to identify any atopic reaction to capsaicin and to illustrate the beneficial effect of capsaicin after taking into account the cooling effect of cream. These applications were repeated 12 hourly. Within 48 hours, there was a marked decrease in the local skin temperature, erythema and local pain in the treated area of $10 \mathrm{~cm}$ in diameter in the right leg compared to the left. Application of the capsaicin cream was then extended to inyolve the whole of the erythematous areas in both legs. This continued to achieve improvement in symptoms and the degree of erythema. Figure 1 shows the legs before and 2 weeks after treatment with capsaicin cream. Capsaicin cream was stopped after 2 months and 6 months later the patient continued to have the symptomatic relief she experienced initially.

\section{Comment}

Erythromelalgia is characterized by burning discomfort, warmth and dermal erythema of the extremities, exacerbation of symptoms by depen- 


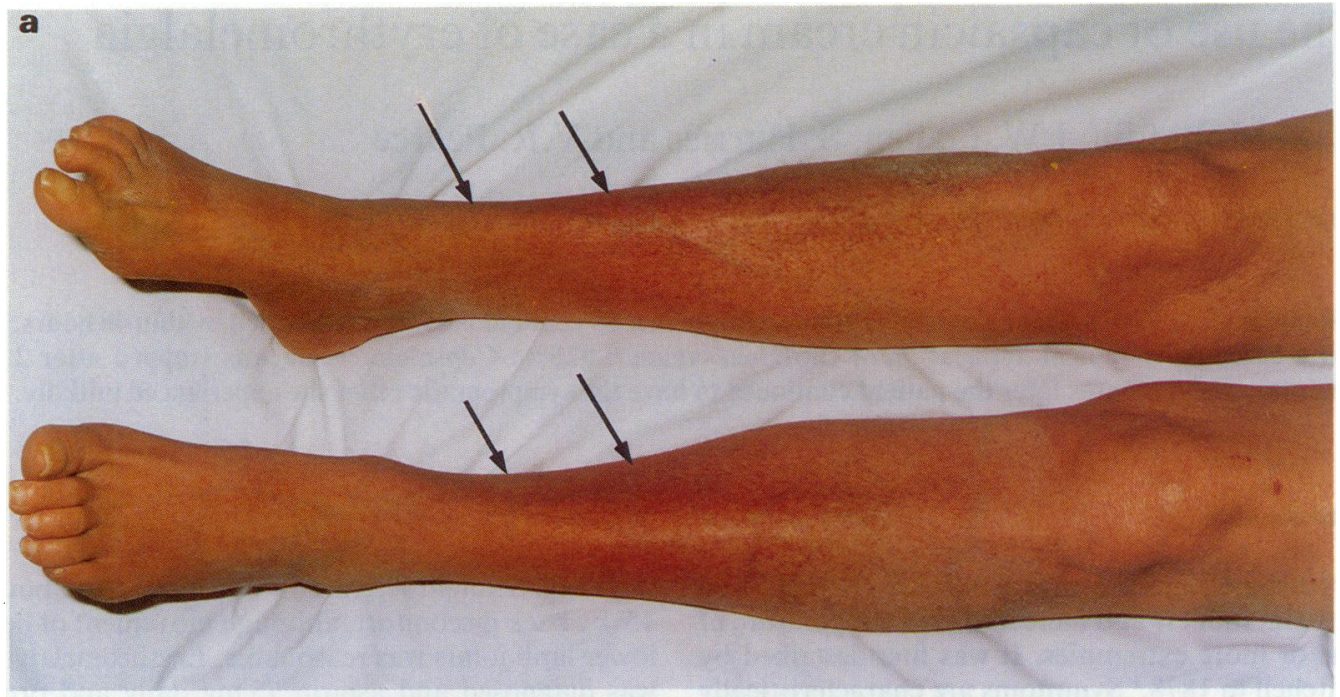

b

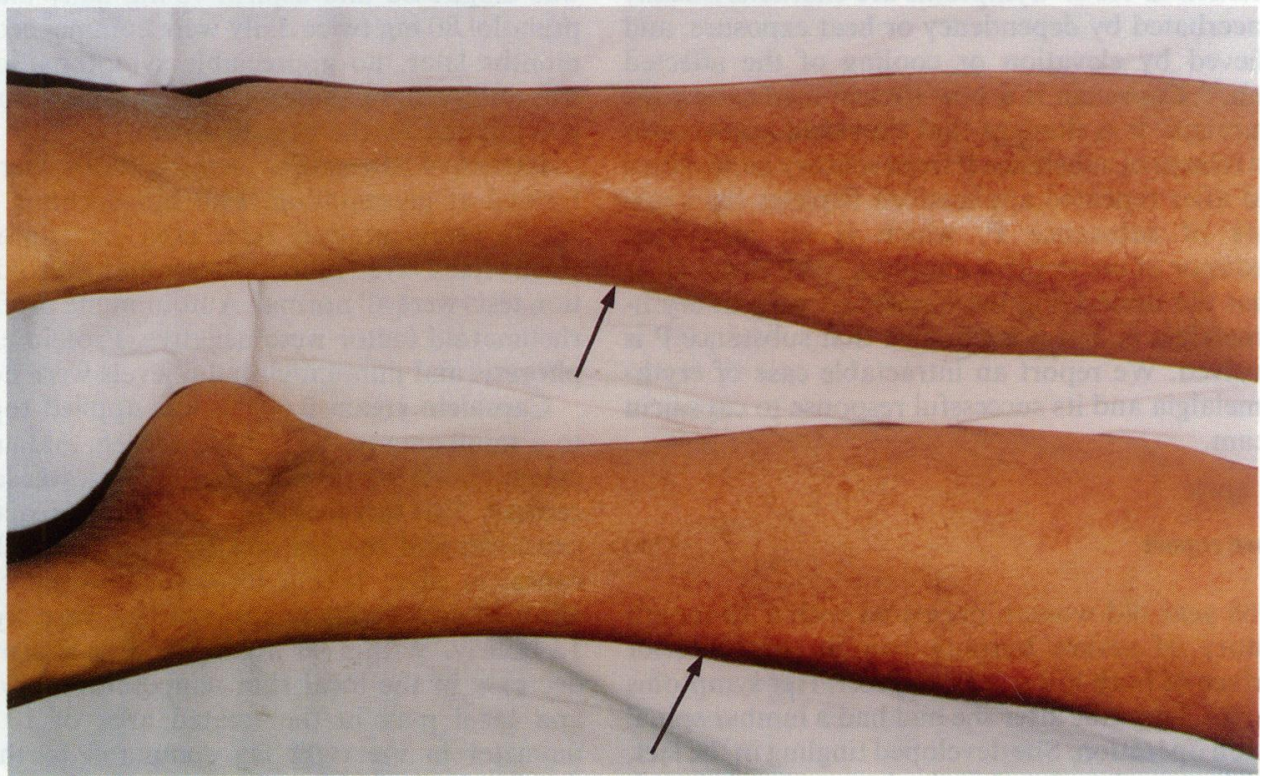

Figure 1 The degree of erythema in the lower limbs before (a) and 2 weeks after (b) treatment with capsaicin cream.

dency or heat exposure, and relief of the discomfort by elevation or cooling of the affected limb. ${ }^{2,3}$ Several types of erythromelalgia have been described, principally an adult-onset and an earlyonset (childhood) form. ${ }^{4}$ The adult-onset type has two subtypes, namely primary (idiopathic) and secondary to an underlying systemic disorder such as myeloproliferative disorder, ${ }^{2}$ hypertension, ${ }^{2}$ diabetes mellitus, ${ }^{2}$ connective tissue disorders, ${ }^{2}$ spinal cord disease ${ }^{5}$ and vasculitis. ${ }^{6}$ On the other hand, the early-onset form usually appears in childhood or adolescence and tends to be idiopathic.

Sugiyama et al. (1991) studied microangiopathically a case of adult onset primary erythromelalgia and demonstrated normal skin sympathetic activity, but no vasoconstriction response was elicited. ${ }^{7}$ In addition, aspirin produced symptomatic relief by activating the skin sympathetic nerve activity and improving the vasoconstriction response. 
Treatment principally is that of the underlying systemic condition in those with secondary erythromelalgia but this is not always possible. However, the majority of patients with erythromelalgia do seem to respond to aspirin. ${ }^{2,3}$ Treatment may be difficult if aspirin has failed and a variety of medications have been tried in those circumstances, for example: indomethacin, ${ }^{3}$ propranolol ${ }^{8}$ and methysergide. ${ }^{9}$

Capsaicin (trans-8-methyl- $N$-vanillyl-6-nonenamide) is a new topical medication for neuralgia. It has been used to treat post-herpetic neuralgia ${ }^{10}$ and painful diabetic peripheral neuropathy ${ }^{11}$ among other painful conditions without the adverse effects associated with systemic medications. In this article we report successful symptomatic relief in erythromelalgia with topical capsaicin, which has to our knowledge never been tried before in this condition. The sensory neurones with unmyelinated C-fibres have been implicated in mediating cutaneous pain. These neurones possess a unique sensitivity to capsaicin, the lipophilicity of which enhances its dissolution and contributes to its

\section{References}

1. Mitchell, S.W. On a rare vasomotor neurosis of the extremities and on maladies with which it may be confounded. Am J Med Sci 1878, 76: 2-36.

2. Babb, R.R., Alarcon-Segovia, D. \& Fairbairn, J.F., II. Erythromelalgia: review of 51 cases. Circulation 1964, 29: 136-141.

3. Michiels, J.J., Abels, J., Steketee, J. et al. Erythromelalgia caused by platelet-mediated arteriolar inflammation and thrombosis in thrombocythemia. Ann Intern Med 1985, 102: 466-471.

4. Kurzrock, R. \& Cohen, P.R. Erythromelalgia: review of clinical characteristics and pathophysiology. Am J Med 1991, 91: 416-422.

5. Collier, J. The occurrence of erythromelalgia in disease of the spinal cord; an account of ten cases. Lancet 1988, 2: 401-440.

6. Ratz, J.L., Bergfeld, W.F. \& Steck, W.D. Erythromelalgia with vasculitis: a review. J Am Acad Dermatol 1979, 1: 443-450.

7. Sugiyama, Y., Hakusui, S., Takahashi, A. et al. Primary erythromelalgia: the role of skin sympathetic activity. Jpn $J$ Med 1991, 30: 564-567. action on sensory neurone membranes. ${ }^{12}$ It is thought that capsaicin accentuates the release of substance $\mathbf{P}$ from sensory nerve fibres and, after repeated applications, depletes the neurones of substance $P$, and hence tachyphylaxis occurs. ${ }^{10}$ Substance $P$ has been shown to be important, if not the essential chemomediators of nociceptive impulses from the periphery to the central nervous system. ${ }^{13.14}$ The effective outcome of capsaicin in this case of erythromelalgia suggests that the symptoms of burning discomfort, warmth and dermal erythema of the extremities are largely mediated by substance $P$. The continued symptomatic relief observed 6 months after cessation of the use of capsaicin cream suggests some changes in the nerve conduction of a damaged peripheral nerve with or without its central connection.

\section{Acknowledgement}

We thank DrS. Rae for allowing us reporting this patient.

8. Bada, J. Treatment of erythromelalgia with propranolol. Lancet 1977 , ii: 412 .

9. Catchpole, B.N. Erythromelalgia. Lancet 1964, i: 909-911.

10. Bernstein, J.E., Bickers, D.R., Dahl, M.V. et al. Treatment of chronic postherpetic neuralgia with topical capsaicin. $\mathbf{J ~ A m}$ Acad Dermatol 1987, 17: 93-96.

11. Capsaicin Study Group. Effect of treatment with capsaicin on daily activities of patients with painful diabetic neuropathy. Diabetes Care 1992, 15: 159-165.

12. Nolte, M.J. Topical capsaicin for postherpetic neuralgia. Drug Intell Clin Pharmacy 1988, 22: 488.

13. Hokfelt, T., Kellerth, J.O., Nilsson, G. et al. Substance P: localization in the central nervous system and in some primary sensory neurons. Science 1975, 190: 889-890.

14. Hokfelt, T., Johansson, O., Ljungdahl, A. et al. Peptidergic neurons. Nature 1980, 284: 515-524. 